\title{
Gentiopicroside Ameliorates Glucose and Lipid Metabolism in T2DM by Activating PI3K/AKT Pathway Via FGFR1
}

\section{Zhanchi Xu}

Guangzhou University of Chinese Medicine

\section{Zeyuan Lin}

Sun Yat-sen University

Jingran Zeng

Sun Yat-Sen University

Rui Chen

Sun Yat-Sen University

Chuting Li

Sun Yat-sen University

\section{Haiming Xiao}

Sun Yat-Sen University

Heqing Huang ( $\square$ huangheq@mail.sysu.edu.cn )

Sun Yat-Sen University https://orcid.org/0000-0002-6135-9901

\section{Shiyue Xu}

Sun Yat-Sen University

\section{Tian Lan}

Guangdong Pharmaceutical University

\section{Study protocol}

Keywords: gentiopicroside, type 2 diabetes mellitus, PI3K/AKT, FGFR1

Posted Date: November 30th, 2021

DOI: https://doi.org/10.21203/rs.3.rs-1085253/v1

License: (c) (i) This work is licensed under a Creative Commons Attribution 4.0 International License. Read Full License 


\section{Abstract}

Background: Abnormalities in lipid and glucose metabolism are are constantly occured in type 2 diabetes (T2DM). However, it can be ameliorated by gentiopicroside (GPS). Considering the key role of fibroblast growth factor receptor 1/phosphatidylinositol 3-kinase/protein kinase B (FGFR1/PI3K/AKT) pathway in T2DM, we explore the possible mechanism of GPS on lipid and glucose metabolism through its effects on FGFR1/PI3K/AKT pathway.

Methods: Palmitic acid (PA)-induced HepG2 cells and a db/db mice were used to clarify the role and mechanism of polydatin on lipid and glucose metabolism.

Results: GPS ameliorated glucose and lipid metabolism disorders in db/db mice and PA-induced HepG2 cells. Furthermore, GPS activated FGFR1/PI3K/AKT pathway including increased the protein expression of FGFR1 and promoted the phosphorylation of PI3K, AKT and Fox01. Additionally, knockdown of FGFR1 reversed the activation of PI3K/AKT pathway by GPS.

Conclusions: The present study demontrates that GPS ameliorates glucose and lipid metabolism disorders via activation of FGFR1/PI3K/AKT pathway.

\section{Background}

Type 2 diabetes mellitus (T2DM) is a metabolic disease with high rate of morbidity and mortality [1]. The number of T2DM patients is increasing at an alarming rate and it has become a global epidemic. However, the exact pathogenesis of T2DM remains unclear, and there is no targeted therapy [2,3]. Therefore, it is urgent to develop more potent drugs.

Glucose and lipid metabolism disorders are the main characteristics of T2DM [4]. Phosphatidylinositol 3kinase/protein kinase $B$ (PI3K/AKT) pathway is a classical pathway that plays the most important role for glucose and lipid metabolism $[5,6]$. After stimulating with receptor tyrosine kinase, the regulatory subunit p85 of PI3K is recruited to cell membrane, and then combines with catalytic subunit p110. Next, p110 catalyzes phosphatidylinositol 4, 5-bisphosphate (PIP2) to PIP3, which binds to the pleckstrin homology domain of AKT and phosphoinositide-dependent kinase 1 (PDK1), thus transfering them to membrane from cytoplasm. As a result, AKT is activated by PDK1 through phosphorylating the sites of Thr 308 and Ser 473 [7]. Forkhead box protein 01 (Fox01) a primary down stream of AKT, is ubiquitously expressed in liver [8]. Activated AKT regulates the protein expressions of key enzymes related to glucose and lipid metabolism such as glucose-6-phosphatase (G6Pase), glucokinase (GCK) and low density lipoprotein receptor (LDLR) via phosphorylating Fox01 $[9,10]$. Therefore, activating PI3K/AKT pathway can ameliorate glucose and lipid metabolism disorders markedly in T2DM.

Fibroblast growth factor 19 (FGF19, the humanortholog of mouse FGF15) is expressed in enterocytes of terminal ileum and secreted to the enterohepatic circulation in response to bile acids via activation of the bile acid nuclear receptor named farnesoid X receptor (FXR) [11]. At present, FGF19 has received 
particular attention owing to its important role in glucose and lipid metabolism [12]. Fibroblast growth factor receptor (FGFR), a receptor tyrosine kinase, mainly consists of FGFR1- FGFR4. FGF19 exerts its function by activating FGFRs [13]. Importantly, the FGFRs is the up-stream of PI3K/AKT pathway. After stimulation, fibroblast growth factor receptor substrate $2 a(F R S 2 a)$ is rapidly tyrosine phosphorylated by FGFRs and functions as conning center to recruits the regulatory subunit p85 of PI3K, eventually activates PI3K/AKT pathway $[14,15]$. However, the protein expression of FGFR is inhibited in T2DM, which may lead to the abnormal PI3K/AKT pathway signal transduction $[16,17]$. Consequently, activating FGFR can improve glucose and lipid metabolism disorders via PI3K/AKT pathway.

Gentiopicroside (GPS) is a nature product with the biological activity of promoting bile acid secretion [18]. Our previous study indicated that GPS could ameliorate glucose and lipid metabolism disorders in T2DM [19]. However, the mechanism was still unclear. Considering the biological activity of GPS in promoting bile acid secretion, we wonder whether GPS ameliorates glucose and lipid metabolism disorders by activating PI3K/AKT pathway via FGFR. The result of network pharmacology showed that FGFR1 was the target of GPS (Supplement Table 1). Further more, GPS had more prominent effect on FGFR1 mRNA expression compared with other subtypes (Supplement Fig. 1). Therefore, we study the effects of GPS on FGFR1/PI3K/AKT pathway to clarify the mechanism of GPS for ameliorating glucose and lipid metabolism disorders. The results of this study showed that GPS effectively ameliorated diabetic glucose and lipid metabolism disorders, and the mechanism was related to the activation of FGFR1/PI3K/AKT pathway.

\section{Methods}

\subsection{Reagents and antibodies}

GPS (used in animal experiment, purity $>98 \%$ ) was obtained from NanJing Dilger Medical Technology Co.,Ltd (Nanjing, China). GPS (used in vitro experiment, purity $>99 \%$ ) was obtained from Nanjing ZheWeiKang Biological Technology Co., Ltd (Nanjing, China). Metformin (Met, used in vitro experiment, purity $>99 \%$ ), methyl thiazolyl tetrazolium (MTT) and insulin were purchased from Beyotime Institute of Biotechnology (Shanghai, China). Met (used in animal experiment) was obtained from American Shanghai Squibb Pharmaceuticals Ltd (Shanghai, China). Dulbecco's modified eagle (DMEM) medium, fetal bovine serum (FBS), trypsin and phosphate buffer solution (PBS) were purchased from Gibico (New York, USA). Palmitic acid (PA), dimethyl sulfoxide (DMSO) and oil red O were purchased from Sigma Aldrich (Missouri, USA). Bovine serum albumin (BSA) was purchased from solarbio (Beijing, China). The detection kits of glycated serum protein (GSP), hemoglobin A1c (HbA1c), triglycerides (TG) and lowdensity lipoprotein cholesterol (LDL-C) were purchased from Jiancheng Bioengineering Institute (Nanjing, China).

Antibody against FGFR1 was obtained from Cell Signaling Technology (Massachusetts, USA). Antibodies against FRS2a, AKT, phosphorylated AKT (p-AKT), GCK and a-Tubulin were purchased from Proteintech Group (Wuhan, China). Antibodies against PI3K and phosphorylated PI3K (p-PI3K) were purchased from 
ABclonal Technology (Massachusetts, USA). Antibodies against Fox01, phosphorylated Fox01 (pFox01), LDLR and Lamine B1 were purchased from Abcam (Massachusetts, USA). Antibody against G6Pase was obtained from Boster (Wuhan, China). Antibody against phosphorylated FRS2a (p-FRS2a) was obtained from Affinity Biosciences (Ohio, USA). GFP antibody was purchased from Beyotime Institute of Biotechnology (Shanghai, China).

\subsection{Animal experiments}

Six-week-old male db/db mice (Animal Quality Certificate Number: 201919681) obtained from Gem Pharmatech Co., Ltd (Nanjing, China) were selected in animal experiments. The mice were kept in specific pathogen free condition and given normal feed. All experiments were conducted in accordance with the China Animal Welfare Legislation and were approved by Ethics Committee on the Care and Use of Laboratory Animals of Sun Yat-sen University (Guangzhou, China).

After adaptation for one week, the $\mathrm{db} / \mathrm{db}$ mice were divided randomly into five groups $(\mathrm{n}=8)$ including diabetic model group, low-dose group (50 mg/kg), medium-dose group (100 mg/kg), high-dose group $(200 \mathrm{mg} / \mathrm{kg})$ and Met group (195 mg/kg) based on fasting blood glucose (FBG) value. Wild $\mathrm{db} / \mathrm{m}(\mathrm{n}=8)$ were the control group. The same volume of normal saline was given to the mice in both control group and diabetic group. Body weight and FBG were measured weekly. After intragastric administration for 10 weeks, all mice were sacrificed to collect the serum. The livers were fixed in $4 \%$ paraformaldehyde or frozen at $-80^{\circ} \mathrm{C}$ for further experiments.

\subsection{Liver tissue staining and immunohistochemistry}

Hematoxylin eosin (HE) staining was conducted as reported method [20]. In short, liver tissues were fixed in $4 \%$ paraformaldehyde at room temperature for more than $24 \mathrm{~h}$. Slices $(5 \mu \mathrm{M})$ were made after being paraffin embedding and then dried at $60^{\circ} \mathrm{C}$ for $24 \mathrm{~h}$. After that, the procedures of deparaffinization, rehydration, hematoxylin and eosin staining were conducted in turn. Finally, the sections were dehydrated and covered with neutral balsam. Meanwhile, periodic acid schiff (PAS) staining and immunohistochemistry were conducted as reported method after paraffin sections were made [21, 22].

Fresh liver tissues were fixed in 4\% paraformaldehyde at room temperature for more than $24 \mathrm{~h}$, and then dehydrated by immersing in $15 \%$ and $30 \%$ sucrose solution. Then optimal cutting temperature compound-embedded slices $(10 \mu \mathrm{M})$ were made and kept at $-20^{\circ} \mathrm{C}$. After that, oil red $\mathrm{O}$ staining was conducted as reported method [23].

\subsection{Cell culture}

HepG2 cells (American Type Culture Collection, Maryland, USA) were cultured with DMEM medium containing $25.5 \mathrm{mM}$ glucose and $10 \%$ fetal serum at $37{ }^{\circ} \mathrm{C}$ in a humidified incubator with $5 \% \mathrm{CO}_{2}$. After serum-deprivation for $12 \mathrm{~h}$, the cells were divided into different groups. Next, HepG2 cells were treated with GPS and $0.25 \mathrm{mM} \mathrm{PA}$ (dissolved in $20 \% \mathrm{BSA}$ ) for $24 \mathrm{~h}$. The cells were stimulated with $100 \mathrm{nM}$ insulin for 10 min before collecting for western blotting. Met was the positive control in this study. 


\subsection{MTT assay}

The cytotoxic of GPS on HepG2 cells was measured by MTT assay. Briefly, HepG2 cells $\left(5 \times 10^{3} \mathrm{cells} / \mathrm{mL}\right.$, $100 \mu \mathrm{L}$ ) were inoculated into 96-well plate. After reaching about $50 \%$ confluence, the cells were cultured with FBS-free DMEM that contained different concentrations of GPS for $24 \mathrm{~h}$. Then $10 \mu \mathrm{L}$ of MTT (5 $\mathrm{mg} / \mathrm{mL}$ ) was added to each well. After $4 \mathrm{~h}$, the supernatant was removed and $200 \mu \mathrm{L}$ of DMSO was added into wells. Finally, the optical density was measured at $570 \mathrm{~nm}$ with microplate reader (Omega BioTek, Georgia, USA).

\subsection{Glucose consumption assay}

Firstly, HepG2 cells were treated with various concentrations of GPS for $24 \mathrm{~h}$. After that, the supernatants were replaced with DMEM without phenol red (New York, USA) containing $100 \mathrm{nM}$ insulin for $4 \mathrm{~h}$. Later, the supernatants were collected to examined glucose content with the method of glucose oxidase (Jiancheng, Nanjing, China). Finally, glucose content was computed by subtracting glucose content in DMEM without phenol red and standardized using the number of cells in each wells (detected by MTT method).

\subsection{Cellular Oil Red O Staining}

Oil red 0 powder was diluted in isopropyl alcohol with the concentration of $0.5 \%$. Then above solution was mixed with distilled water by 3:2 and filtered to remove residual particles. After washing with PBS for 3 times, HepG2 cells were fixed with $4 \%$ paraformaldehyde for $10 \mathrm{~min}$ and then recolored with oil red 0 dye liquor for $30 \mathrm{~min}$ at room temperature. Finally, the cells were observed by cell imaging system (EVOS FL Auto, Thermo Fisher Scientific, Massachusetts, USA) after hematoxylin staining for 5 min.

\subsection{Western blot}

The liver tissues or HepG2 cells were lysed with RIPA lysis buffer (Beyotime Institute of Biotechnology, Shanghai, China) mixed with protease and phosphatase inhibitor cocktail (Selleck Chemicals, Texas, USA). Nuclear and cytoplasmic proteins were extracted by special protein extraction kit (Active Motif, Carlsbad, USA). Later, the concentration of protein was measured by BCA Protein Assay Kit (Pierce, Illinois, USA). After that, equal amount of proteins boiled with loading buffer for 5 min were subjected to $8-12 \%$ sodium dodecyl sulfate polyacrylamide gel electrophoresis (SDS-PAGE) and transferred to polyvinylidene difluoride (PVDF) membranes (Mellipore, Massachusetts, USA). After sealing with 5\% skim milk at room temperature for $1 \mathrm{~h}$, PVDF membranes were incubated with primary antibodies and secondary antibodies labeled with HRP (Promega, Wisconsin, USA). The signals of antibodies were visualized with ECL detection kit (Pierce, Illinois, USA) and observed by Tanon 5200 Chemiluminescent Imaging System (Shanghai Tanon Technology Co., Ltd., Shanghai, China). The software of Image J was applied for quantitative analysis.

\subsection{Immunofluorescence}


HepG2 cells were cultured on glass coverslips placed in 24-well plate. After intervention, the cells were fixed in $4 \%$ paraformaldehyde for $10 \mathrm{~min}$ and permeabilized with $0.1 \%$ Triton X-100 (dissolved in PBS) for 10 min successively. Next, the cells were blocked with $10 \%$ goat serum (Boster Biological Technology Co., Ltd., Wuhan, China) for $1 \mathrm{~h}$ at room temperature and incubated with primary antibody at $4{ }^{\circ} \mathrm{C}$ for $24 \mathrm{~h}$. After that, HepG2 cells were incubated with secondary antibody (Thermo, Massachusetts, USA) and DAPI (Beyotime Institute of Biotechnology, Shanghai, China). Finally, the fluorescent images were captured by Zeiss LSM 510 laser confocal fluorescence microscope (Oberkochen, Germany).

\subsection{Immunoprecipitation assay}

The proteins were extracted by lysing HepG2 cells with immunoprecipitation (IP) buffer (Beyotime Institute of Biotechnology, Shanghai, China) containing protease inhibitor (Selleck Chemicals, Texas, USA) on ice for $30 \mathrm{~min}$. Next, $400 \mu \mathrm{g}$ proteins were incubated with $20 \mu \mathrm{L}$ protein agarose $\mathrm{A} / \mathrm{G}$ beads (Thermo, Massachusetts, USA) to reduce the nonspecific combination, followed by collecting the supernatants with transient centrifugation. Later, the supernatants were incubated with antibodies (against FGFR1 or IgG) with shaking for $12 \mathrm{~h}$ at $4{ }^{\circ} \mathrm{C}$. Subsequently, protein agarose A/G beads $(20 \mu \mathrm{L})$ were added to the supernatants for further incubation $\left(4^{\circ} \mathrm{C}, 4 \mathrm{~h}\right)$. Collecting beads by transient centrifugation and washing beads with IP buffer for 3 times. Finally, $20 \mu \mathrm{L}$ loading buffer (Fdbio, Hangzhou, China) was added and the mixtures were boiled for 5 min to denature proteins. The protein levels were detected according to the method of western blot.

\subsection{Transfections of plasmids and small-interfering RNA}

FGFR1 plasmid was proved by Shanghai Genechem Co.,Ltd. (Shanghai, China) and transfected according to instruction of LTX reagent and PLUS ${ }^{\text {TM }}$ reagent (Life Technologies, New York, USA).

The FGFR1 siRNA oligonucleotides was purchased from GenePharma (Shanghai, China). The sequences are as follows:

sense: 5'-CGGUCAUCGUCUACAAGAUTT-3';

antisense: 5'-AUCUUGUAGACGAUGACCGTT-3'.

The transfection of siRNA was conducted according to the instruction of Lipofectamine RNAiMAX reagent (Life Technologies, New York, USA).

\subsection{Statistical analysis}

All experimental data were analyzed by the software of Graphpad Prism 5.0 and expressed as mean \pm standard deviation (SD). The experiments in this study were performed at least three times with similar results. Unpaired Student's test was adopted to analyse the data between two groups. For multiple comparisons, one-way ANOVA with post hoc was performed. A value of $P<0.05$ was considered as statistically significant. 


\section{Results}

\subsection{GPS attenuates metabolic disorder in $\mathrm{db} / \mathrm{db}$ mice}

The $\mathrm{db} / \mathrm{db}$ mice were used to assess the protective effects of GPS on T2DM in our current study. After 10week GPS treatment, blood biochemical parameters of $\mathrm{db} / \mathrm{db}$ mice were analyzed and the liver tissues were collected for further study (Fig. 1a). The results showed that GPS remarkably attenuated the symptoms of polydipsia, polyphagia and polyuria in diabetic mice (Fig. 1b, c and d). In addition, body weight was significantly decreased by GPS treatment compared with diabetic group (Fig. 1e).

Next, the increased level of FBG in diabetic mice significantly reduced by GPS treatment (Fig. 1f). Furthermore, GSP and HbA1c, another two representative biomarkers to assess the long-term diabetic complications were analyzed. GPS treatment also significantly decreased the levels of GSP and HbA1c (Fig. 1g, h). Additionally, GPS treatment down-regulated the levels of serum TG and LDL-C in diabetic mice (Fig. 1i, j). Taken together, GPS improved glucose and lipid metabolism disorders in T2DM mice.

\subsection{GPS attenuates glucose and lipid metabolic disorder in the livers of $\mathrm{db} / \mathrm{db}$ mice}

The disorders of glucose and lipid metabolism in T2DM is mainly caused by the elevated hepatic gluconeogenesis, impaired hepatic glycogen synthesis and excessive hepatic lipid accumulation [24, 25]. Thus, the effects of GPS on glucose and lipid metabolism in liver were examined. The results showed that glycogen storage was decreaed in diabetic mouse livers. However, glycogen storage were increased by GPS treatment. Oil Red 0 staining showed excessive lipid was accumulated in diabetic mouse liver compared to the control group, whereas significantly alleviated by GPS treatment (Fig. 2a and Supplement Fig. 2a ).

G6Pase is a rate-limiting enzyme of hepatic gluconeogenesis, which is closly related to blood glucose. Over-activation of G6Pase contributes to the increased export of hepatic glucose [26]. GCK, a key enzyme for glycolysis and glycogen synthesis, also plays vital role in the metabolism of glucose [27]. LDLR is a cell-surface protein mediating LDL transport. The functional deficiency of LDLR is one of the main causes leading to hepatic lipid accumulation [28]. Considering the key roles of G6Pase, GCK and LDLR in glucose and lipid metabolism, we further detected the effects of GPS on above proteins.

Western blot assay showed that GPS up-regulated the protein expressions of GCK and LDLR but inhibited the protein expression of G6Pase (Fig. 2b), which were consistent with the results of immunohistochemistry (Fig. 2c and Supplement Fig. 2b, c and d). Taken together, these data demonstrated that GPS meliorated hepatic glucose and lipid metabolism disorder in diabetic mice.

\subsection{GPS activated FGFR1/PI3K/AKT pathway in the liver of $\mathrm{db} / \mathrm{db}$ mice}


Fox01 is a typical transcriptional factor related to protein expressions of LDLR, GCK as well as G6Pase. The transcriptional activity of FoxO1 is inhibited by the activated PI3K/AKT signaling. It is well-known that GPS regulates the expressions of LDLR, GCK and G6Pase. However, whether GPS activates $\mathrm{PI} 3 \mathrm{~K} / \mathrm{AKT} / \mathrm{Fox} 01$ pathway is still unclear. The results of western blot showed that the levels of $\mathrm{p}-\mathrm{PI} 3 \mathrm{~K}, \mathrm{p}-$ AKT and p-FoxO1 were markedly decreased in diabetic group, whereas restored by GPS treatment (Fig. 3a).

FGFR1, the potential target for the treatment of T2DM can active PI3K/Akt pathway after interacting with FRS2a. To further clarify the mechanism of GPS activating PI3K/AKT pathway, the protein expressions of FGFR1 and FRS2 $a$ were detected. Compared with the normal group, the levels of FGFR1, p-FRS2 $a$ and FRS2a were decreased. However, GPS treatment markedly up-regulated the protein expressions of FGFR1, p-FRS2 $a$ and FRS2a (Fig. 3b, c and Supplement Fig. 2e, f) and Met had no effect on FGFR1 expression. In conclusion, GPS activates FGFR1/PI3K/AKT pathway in the liver of diabetic mice.

\subsection{GPS ameliorated glucose and lipid metabolism disorders in PA-induced HepG2 cells}

To further confirm the role and mechanism of GPS in the improvement of glucose and lipid metabolism disorders, we established the model of glucose and lipid metabolism disorders in vitro [23].

As shown in Fig. 4a, GPS with the concentration less than $320 \mu \mathrm{M}$ showed no cytotoxicity to HepG 2 cells. Further more, the results of preliminary dose test suggested that GPS promoted glycogen synthesis and glucose consumption starting at $20 \mu \mathrm{M}$ (Supplement Fig. $2 \mathrm{~g}$ and h). Thus, the concentrations of 20,40 and $80 \mu \mathrm{M}$ were selected in next studies.

The level of glycogen and and glucose consumption decreased in PA-induced HepG2 cells. GPS treatment significantly induced glycogen storage (Fig. 4b) and glucose consumption (Fig. 4c) which were consistent with the results determined by PAS staining (Fig. 4d). In addition, Oil Red 0 staining showed that GPS treatment reduced lipid accumulation in PA-induced HepG2 cells (Fig. 4e). Moreover, GPS treatment up-regulated the protein levels of GCK and LDLR but inhibited the protein expression of G6Pase (Fig. 4f). These results confirmed that GPS attenuates glucose and lipid metabolism disorders in vivo.

\subsection{GPS activated PI3K/AKT pathway in PA-induced HepG2 cells}

We further investigated the effects of GPS on PI3K/AKT pathway in PA-induced HepG2 cells. The results showed the levels of p-PI3K, p-AKT and p-Fox01 were decreased, while the treatment of GPS significantly increased the expressions of p-PI3K, p-AKT and p-FoxO1 (Fig. 5a). In addition, we detected the levels of FoxO1 in the nuclear and cytoplasmic and found that the nuclear protein level of FoxO1 was elevated in PA-induced HepG2 cells. However, the distribution of FoxO1 in nuclear decreased after the treatment of GPS (Fig. $5 b$ and c). Immunofluorescence assays also showed that GPS prevented FoxO1 translocation into the nucleus (Fig. 5d). 


\subsection{GPS up-regulated FGFR1 protein expression in PA- induced HepG2 cells}

We further studied the effects of GPS on FGFR1 and FRS2 $a$ in vivo. The results showed that the protein levels of FGFR1 and FRS2a significantly decreased in a time-dependent manner when HepG2 cells were stimulated with PA (Fig. 6a). Furthermore, FGFR1 over-expression activated PI3K/AKT pathway and ameliorated glucose and lipid metabolism disorders in PA-induced HepG2 cells (Supplement Fig. 3 and Supplement Fig. 4). Therefore, we conclude that down-regulated expression of FGFR1 is one of the key factors leading to the inhibition of PI3K/AKT pathway, which eventually leads to the disorders of glucose and lipid metabolism.

Importantly, the protein levels of FGFR1 and FRS2a were efficiently increased after the intervention of GPS (Fig. 6b, c). However, Met had on effect on FGFR1 expression. Additionally, IP assay suggested that FGFR1 interacted with FRS2 $a$ in normal condition, and the interaction decreased after PA stimulation. However, the treatment of GPS significantly promoted the bind of FGFR1 with FRS2a (Fig. 6d).

\subsection{Knockdown of FGFR1 reversed the avtivation of PI3K/AKT pathway by GPS in HepG2 cells cultured by PA}

According to the experiments in vitro and in vivo, we concluded that GPS ameliorated glucose and lipid metabolism disorders via activating FGFR1/PI3K/AKT pathway. To further clarify the significance of FGFR1 in this process, we carried out validation experiments after the HepG2 cells were transfected with siRNA oligonucleotides targeting FGFR1.

The protein level of FGFR1 was efficiently depleted after HepG2 cells were transfected with siRNA oligonucleotides targeting FGFR1 (Fig. 7a). Compared to GPS treatment group, the GCK as well as LDLR protein levels were up-regulated and G6Pase protein expression was decreased when GPS and siRNA oligonucleotides targeting FGFR1 were given at the same time (Fig. 7b). Moreover, the knockdown of FGFR1 reversed the effect GPS on the level of p-Fox01 (Fig. 7c). Meanwhile, knockdown of FGFR1 reversed the down-regulated effect of GPS on the nuclear accumulation of FoxO1 (Fig. 7e, f). In addition, the activatory effects of GPS on p-FRS2a, p-PI3K and p-AKT abrogated after the knockdown of FGFR1 (Fig. 8a, b).

In conclusion, these results further confirm that GPS ameliorate glucose and lipid metabolic disorder in T2DM, at least in part, via activation of FGFR1/PI3K/AKT pathway (Fig. 8c).

\section{Discussion}

The number of diabetes patients in the world has reached 415 million and is expected to increase to 642 million by 2040 [29]. T2DM is the main type of diabetes. Lipid and glucose metabolism disorders are the primary pathological characteristics of T2DM. Moreover, it is also regarded as the basic pathology in 
diabetic complications [30]. Therefore, glycaemic control and management of hyperlipidaemia is the primary strategy in T2DM treatment, throughout the whole process of T2DM therapy.

Elevated hepatic gluconeogenesis, impaired hepatic glycogen synthesis and excessive hepatic lipid accumulation are the main cause of lipid and glucose metabolism disorders. Moreover, reversing above pathological changes in liver is of great importance for T2DM treatment. The results of this study showed that GPS improved serum parameters of T2DM mice including down-regulating the levels of TG, LDL-C, FBG, GSP and HbA1c. Next, we detected the pathological changes in liver. The results indicated that GPS inhibited hepatic gluconeogenesis and lipid accumulation as well as promoted hepatic glycogen synthesis. Similarly, GPS ameliorated glucose and lipid metabolism disorders in PA-induced HepG2 cells.

As a rate-limiting enzyme of gluconeogenesis, G6Pase is closly related to glycometabolism. A large number of studies have confirmed that the expression of G6Pase is sharply increased in diabetic mice [10]. However, G6Pase knockout mice exhibit increased glycogen accumulation [31]. Besides that, inhibiting G6Pase expression involved in the improvement of glucose metabolism disorder in T2DM. GCK, one of a key enzymes for the regulation of glycolysis and glycogen synthesis, can catalyze the phosphorylation of glucose to glucose to 6-phosphate (G6P). Decreased GCK expression contributes to the impaired glycogen synthase and glycolysis. LDLR is a membrane protein on the cell surface that has a central role in cholesterol homeostasis. Down-regulated expression of LDLR is recognized as the key factor responsible for hypercholesterolemia. Mounting clinical evidences have showed that inhibiting the down-regulation of LDLR could alleviate the hypercholesterolemia in T2DM. Consequently, reversing the changes of G6Pase, GCK and LDLR in liver can be effective ameliorate glucose and lipid metabolism disorders in T2DM. Based on this, the effects of GPS on G6Pase, GCK and LDLR were further studied. The results suggested that GPS notably suppresed the changes of G6Pase, GCK and LDLR in liver of $d b / d b$ mice and PA-induced HepG2 cells.

At present, it is believed that the abnormal state of PI3K/AKT pathway is the most important factor leading to glucose and lipid metabolism disorders. More importantly, activated PI3K/AKT pathway can influence the protein expressions of G6Pase, GCK and LDLR via Fox01, eventually improve glucose and lipid metabolism disorders. Based on the fact that GPS suppresed the changes of G6Pase, GCK and LDLR, we further studied the effects of GPS on PI3K/AKT/Fox01 pathway. All results in vitro and in vivo experiments showed that GPS effectively actived PI3K/AKT/Fox01 pathway.

FGF1, FGF19 and FGF21, the recently described members of the FGF family, have notable regulatory effects on carbohydrate and lipid metabolism [32,33]. FGFs exert their function by binding to FGFRs. Binding of FGFs to the extracellular receptor domain can induce the phosphorylation of cytoplasmic tyrosine residue and activate the intracellular domain, eventually couple the receptor to intracellular signal transduction pathways. More importantly, the FGFs/FGFRs signaling is the up-stream of PI3K/AKT pathway. After FGFs stimulation, FRS2a is rapidly tyrosine phosphorylated by FGFRs and functions as conning center for the formation of the FRS2a-GRB2-GAB1 complex. Above complex further recruits p85, then activates PI3K/AKT pathway [15]. So far, four genes encoded FGFRs (FGFR1-FGFR4) have been 
reported. However, FGF21 particularly interact with its receptor subtype 1 [13]. FGF1-induced glucoselowering is mediated by FGFR1[34]. Although FGF19 exerts its function mainly by FGFR4, it can signal through FGFR1 as well [13]. Consequently, FGFR1 is considered as the most potential target for the treatment of metabolic syndromes including T2DM [35]. Currently, the drug development about FGFR1 mainly focus on FGF analogues. Owing to the side effects of growth factor such as liver mitogenicity of FGF19 and bone loss of FGF21, the drug development of FGFs is limited [11]. The small molecule targeting FGFR1 may help the development of safe and effective drugs to treat T2DM. Our study found that GPS could active PI3K/AKT pathway via FGFR1, which showed a certain difference compared with the therapeutic drugs for T2DM such as Met. GPS, a natural product mainly isolated from Gentianae Radix et Rhizoma is reported to improve glucose and lipid metabolism disorders in our previous study. We further explore specific mechanism in this study. The present study lay the foundation for clinical application of GPS for T2DM treatment.

\section{Conclusions}

In conclusion, these results further confirm that GPS ameliorate glucose and lipid metabolic disorder in T2DM, at least in part, via activation of FGFR1/PI3K/AKT pathway.

\section{Abbreviations}

AKT, protein kinase B; BSA, Bovine serum albumin; DMEM, Dulbecco's modified eagle; DMSO, dimethyl sulfoxide; FBG, fasting blood glucose; FBS, fetal bovine serum; FGF19, Fibroblast growth factor 19; FGFR, Fibroblast growth factor receptor; Fox01, forkhead box protein 01; FRS2a, fibroblast growth factor receptor substrate 2a; FXR, farnesoid X receptor; GCK, glucokinase; G6Pase, glucose-6-phosphatase; GPS, gentiopicroside; GSP, glycated serum protein; HbA1c, hemoglobin A1c; HE, Hematoxylin eosin; IP, immunoprecipitation; LDL-C, low-density lipoprotein cholesterol; LDLR, low density lipoprotein receptor; Met, Metformin; MTT, methyl thiazolyl tetrazolium; PA, palmitic acid; p-AKT, phosphorylated AKT; PAS, periodic acid schiff; PBS, phosphate buffer solution; PDK1, phosphoinositide-dependent kinase 1; $p$ Fox01, phosphorylated Fox01; p-FRS2a, phosphorylated FRS2a; PIP2, phosphatidylinositol 4, 5bisphosphate; p-PI3K, phosphorylated PI3K; PVDF, polyvinylidene difluoride; T2DM, type 2 diabetes mellitus; TG, triglycerides;SD, standard deviation; SDS-PAGE, sodium dodecyl sulfate polyacrylamide gel electrophoresis;

\section{Declarations}

\section{Acknowledgements}

The work was supported by the National Natural Science Foundation of China (No. 81770816 and 81973375) and Research and Development Projects in Key Areas of Guangdong Province (No:

2020B1111100004). We gratefully thank Dr. Yan Yang (Sun Yat-sen University) and Xiaohong Sun (Sun Yat-sen University) for taking time to edit and critique the manuscript. 


\section{Authors' contributions}

Zhanchi Xu: Conceptualization, Methodology, Software, Validation, Formal analysis, Data Curation, Writing - Original Draft, Visualization. Zeyuan Lin: Conceptualization, Methodology, Software, Validation, Formal analysis, Writing-Review \& Editing, Visualization. Jingran Zeng: Software, Validation, Formal analysis, Writing-Review \& Editing, Visualization. Rui Chen, Chuting Li and Haiming Xiao: Investigation, Writing - Review \& Editing. Heqing Huang: Conceptualization, Data Curation, Writing - Review \& Editing, Supervision, Project administration, Funding acquisition. Shiyue Xu and Tian Lan: Conceptualization, Data Curation, Writing - Review \& Editing, Supervision.

\section{Author details}

${ }^{1}$ School of Pharmaceutical Sciences, Sun Yat-sen University, Guangzhou 510006, China

${ }^{2}$ School of Pharmaceutical Science, Guangzhou University of Chinese Medicine, Guangzhou 510006, China

${ }^{3}$ Guangdong Pharmaceutical University , Guangzhou 510006, China

${ }^{4}$ Department of Hypertension and Vascular Disease, The First Affiliated Hospital, Sun Yat-sen University, Guangzhou, 510080, China

\section{Funding}

The work was supported by the National Natural Science Foundation of China (No. 81770816 and 81973375) and Research and Development Projects in Key Areas of Guangdong Province (No: 2020B1111100004).

\section{Competing interests}

The authors declare that there are no competing interests associated with the manuscript.

\section{Availability of data and materials}

Data and study materials are available.

\section{Consent for publication}

Authors give the full consent to publish the present article.

\section{Ethics approval and consent to participate}

All experimental procedures were carried out in accordance with the China Animal Welfare Legislation, and approved by the Ethics Committee on the Care and Use of Laboratory Animals of Sun Yat-sen University. 


\section{References}

1. Yun J-S, Ko S-H: Current trends in epidemiology of cardiovascular disease and cardiovascular risk management in type 2 diabetes. Metabolism 2021, 123:154838.

2. Siegel KR, Albright AL: Population-level approaches to preventing type 2 diabetes globally. Endocrinology and Metabolism Clinics of North America 2021, 50(3):401-414.

3. Gouda P, Zheng S, Peters T, Fudim M, Randhawa VK, Ezekowitz J, Mavrakanas TA, Giannetti N, Tsoukas M, Lopes $\mathrm{R}$ et al: Clinical phenotypes in patients with type 2 diabetes mellitus: characteristics, cardiovascular outcomes and treatment strategies. Current Heart Failure Reports 2021, 18(5):253-263.

4. Wang Y, Ye J, Li J, Chen C, Huang J, Liu P, Huang H: Polydatin ameliorates lipid and glucose metabolism in type 2 diabetes mellitus by downregulating proprotein convertase subtilisin/kexin type 9 (PCSK9). Cardiovascular Diabetology 2016, 15(1).

5. Gong X, Xiong L, Bi C, Zhang B: Diosmetin ameliorate type 2 diabetic mellitus by up-regulating corynebacterium glutamicum to regulate IRS/PI3K/AKT-mediated glucose metabolism disorder in KK-Ay mice. Phytomedicine 2021, 87:153582.

6. Zheng X-D, Huang Y, Li H: Regulatory role of apelin-13-mediated PI3K/AKT signaling pathway in the glucose and lipid metabolism of mouse with gestational diabetes mellitus. Immunobiology 2021, 226(5):152135.

7. Zhang Z, Liu H, Liu J: Akt activation: a potential strategy to ameliorate insulin resistance. Diabetes Research and Clinical Practice 2019, 156:107092.

8. Wu F, Shao Q, Xia Q, Hu M, Zhao Y, Wang D, Fang K, Xu L, Zou X, Chen Z et al: A bioinformatics and transcriptomics based investigation reveals an inhibitory role of Huanglian-Renshen-Decoction on hepatic glucose production of T2DM mice via PI3K/Akt/FoxO1 signaling pathway. Phytomedicine 2021, 83:153487.

9. Chen L, Sun X, Xiao H, Xu F, Yang Y, Lin Z, Chen Z, Quan S, Huang H: PAQR3 regulates phosphorylation of FoxO1 in insulin-resistant HepG2 cells via NF-KB signaling pathway. Experimental Cell Research 2019, 381(2):301-310.

10. Pitaloka DMIFA, Ko C-H, Lin M-T, Yeh S-L, Yeh C-L: Glutamine administration promotes hepatic glucose homeostasis through regulating the PI3K/AKT pathway in high-fat diet-induced obese mice with limb ischemia. Nutrition Research 2019, 68:45-53.

11. Babaknejad N, Nayeri H, Hemmati R, Bahrami S, Esmaillzadeh A: An overview of FGF19 and FGF21: the therapeutic role in the treatment of the metabolic disorders and obesity. Hormone and Metabolic Research 2018, 50(06):441-452.

12. Talukdar S, Kharitonenkov A: FGF19 and FGF21: in NASH we trust. Molecular Metabolism 2021, 46:101152.

13. Henriksson E, Andersen B: FGF19 and FGF21 for the treatment of NASH-two sides of the same coin? differential and overlapping effects of FGF19 and FGF21 from mice to human. Frontiers in 
Endocrinology 2020, 11.

14. Barylko B, Chen Y-J, Hennen J, Angert I, Chen Y, Mueller JD, Sun H-Q, Taylor CA, Liou J, Yin H et al: Myristoylation-dependent palmitoylation of the receptor tyrosine kinase adaptor FRS2a.

Biochemistry 2019, 58(25):2809-2813.

15. Feng L, Zhang H H, Wang W, Zheng J, Chen D B: Compartmentalizing proximal FGFR1 signaling in ovine placental artery endothelial cell caveolae1. Biology of Reproduction 2012, 87(2).

16. Zhou Y, Li C, Wang X, Deng P, He W, Zheng H, Zhao L, Gao H: Integration of FGF21 signaling and metabolomics in high-fat diet-induced obesity. Journal of Proteome Research 2021, 20(8):39003912.

17. Martínez-Fernández L, González-Muniesa P, Sáinz N, Laiglesia LM, Escoté X, Martínez JA, MorenoAliaga MJ: Maresin 1 regulates hepatic FGF21 in diet-induced obese mice and in cultured hepatocytes. Molecular Nutrition \& Food Research 2019, 63(24):1900358.

18. Tang X, Yang Q, Yang F, Gong J, Han H, Yang L, Wang Z: Target profiling analyses of bile acids in the evaluation of hepatoprotective effect of gentiopicroside on ANIT-induced cholestatic liver injury in mice. Journal of Ethnopharmacology 2016, 194:63-71.

19. Xiao H, Sun X, Liu R, Chen Z, Lin Z, Yang Y, Zhang M, Liu P, Quan S, Huang H: Gentiopicroside activates the bile acid receptor Gpbar1 (TGR5) to repress NF-kappaB pathway and ameliorate diabetic nephropathy. Pharmacological Research 2020, 151:104559.

20. Zhang H, Hui J, Yang J, Deng J, Fan D: Eurocristatine, a plant alkaloid from Eurotium cristatum, alleviates insulin resistance in $\mathrm{db} / \mathrm{db}$ diabetic mice via activation of PI3K/AKT signaling pathway. European Journal of Pharmacology 2020, 887:173557.

21. Sawai K, Mukoyama M, Mori K, Yokoi H, Koshikawa M, Yoshioka T, Takeda R, Sugawara A, Kuwahara T, Saleem MA et al: Redistribution of connexin43 expression in glomerular podocytes predicts poor renal prognosis in patients with type 2 diabetes and overt nephropathy. Nephrology Dialysis Transplantation 2006, 21(9):2472-2477.

22. Zhou B-H, Yang J-Y, Ding H-Y, Chen Q-P, Tian E-J, Wang H-W: Anticoccidial effect of toltrazuril and Radix Sophorae Flavescentis combination: reduced inflammation and promoted mucosal immunity. Veterinary Parasitology 2021, 296:109477.

23. Xu F, Xiao H, Liu R, Yang Y, Zhang M, Chen L, Chen Z, Liu P, Huang H: Paeonol Ameliorates glucose and lipid metabolism in experimental diabetes by activating AKT. Frontiers in Pharmacology 2019, 10.

24. Dong R, Yang X, Wang C, Liu K, Liu Z, Ma X, Sun H, Huo X, Fu T, Meng Q: Yangonin protects against non-alcoholic fatty liver disease through farnesoid $X$ receptor. Phytomedicine 2019, 53:134-142.

25. Zheng T, Yang X, Wu D, Xing S, Bian F, Li W, Chi J, Bai X, Wu G, Chen X et al: Salidroside ameliorates insulin resistance through activation of a mitochondria-associated AMPK/PI3K/AKT/GSK3 $\beta$ pathway. British Journal of Pharmacology 2015, 172(13):3284-3301.

26. Jiang Z, Zhou J, Li T, Tian M, Lu J, Jia Y, Wan G, Chen K: Hepatic deficiency of poldip2 in type 2 diabetes modulates lipid and glucose homeostasis. Metabolism 2019, 99:90-101. 
27. Matschinsky FM, Wilson DF: The central role of glucokinase in glucose homeostasis: a perspective 50 years after demonstrating the presence of the enzyme in islets of langerhans. Frontiers in Physiology 2019, 10.

28. Momtazi-Borojeni AA, Jaafari MR, Abdollahi E, Banach M, Sahebkar A, Zhao R: Impact of PCSK9 immunization on glycemic indices in diabetic rats. Journal of Diabetes Research 2021, 2021:1-11.

29. Standl E, Khunti K, Hansen TB, Schnell O: The global epidemics of diabetes in the 21 st century: current situation and perspectives. European Journal of Preventive Cardiology 2019, 26(2_suppl):714.

30. Zoja C, Xinaris C, Macconi D: Diabetic nephropathy: novel molecular mechanisms and therapeutic targets. Frontiers in Pharmacology 2020, 11.

31. Salganik SV, Weinstein DA, Shupe TD, Salganik M, Pintilie DG, Petersen BE: A detailed characterization of the adult mouse model of glycogen storage disease la. Laboratory Investigation 2009, 89(9):1032-1042.

32. Lin Q, Huang Z, Cai G, Fan X, Yan X, Liu Z, Zhao Z, Li J, Li J, Shi H et al: Activating adenosine monophosphate-activated protein kinase mediates fibroblast growth factor 1 protection from nonalcoholic fatty liver disease in mice. Hepatology 2021, 73(6):2206-2222.

33. Kharitonenkov A: FGFs and metabolism. Current Opinion in Pharmacology 2009, 9(6):805-810.

34. Gasser E, Moutos CP, Downes M, Evans RM: FGF1- a new weapon to control type 2 diabetes mellitus. Nature Reviews Endocrinology 2017, 13(10):599-609.

35. Boehm M, Crawford M, Moscovitz JE, Carpino PA: Diabetes area patent participation analysis-part II: years 2011-2016. Expert Opinion on Therapeutic Patents 2017, 28(2):111-122.

\section{Figures}


i.g. $0.1 \mathrm{~mL} / 10 \mathrm{~g}$

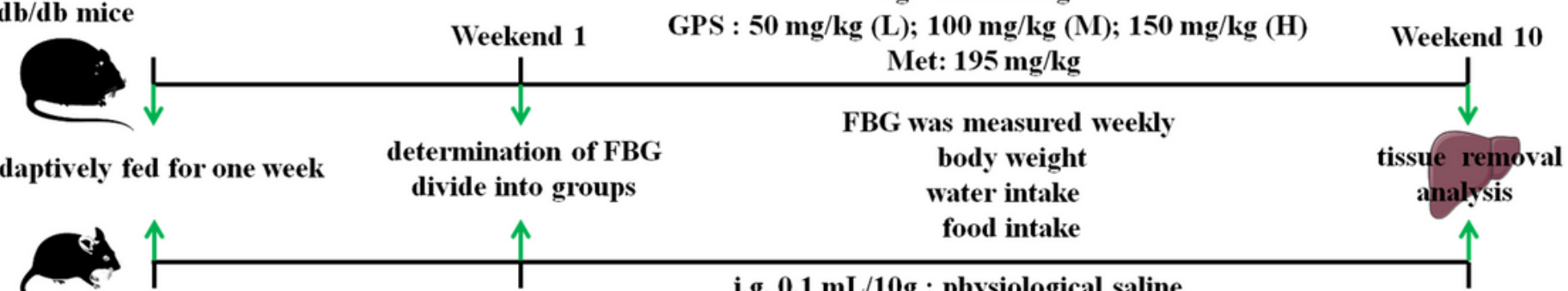

$\mathbf{d b} / \mathbf{m}$

\section{Weekend 1}

i.g. $0.1 \mathrm{~mL} / 10 \mathrm{~g}$; physiological saline
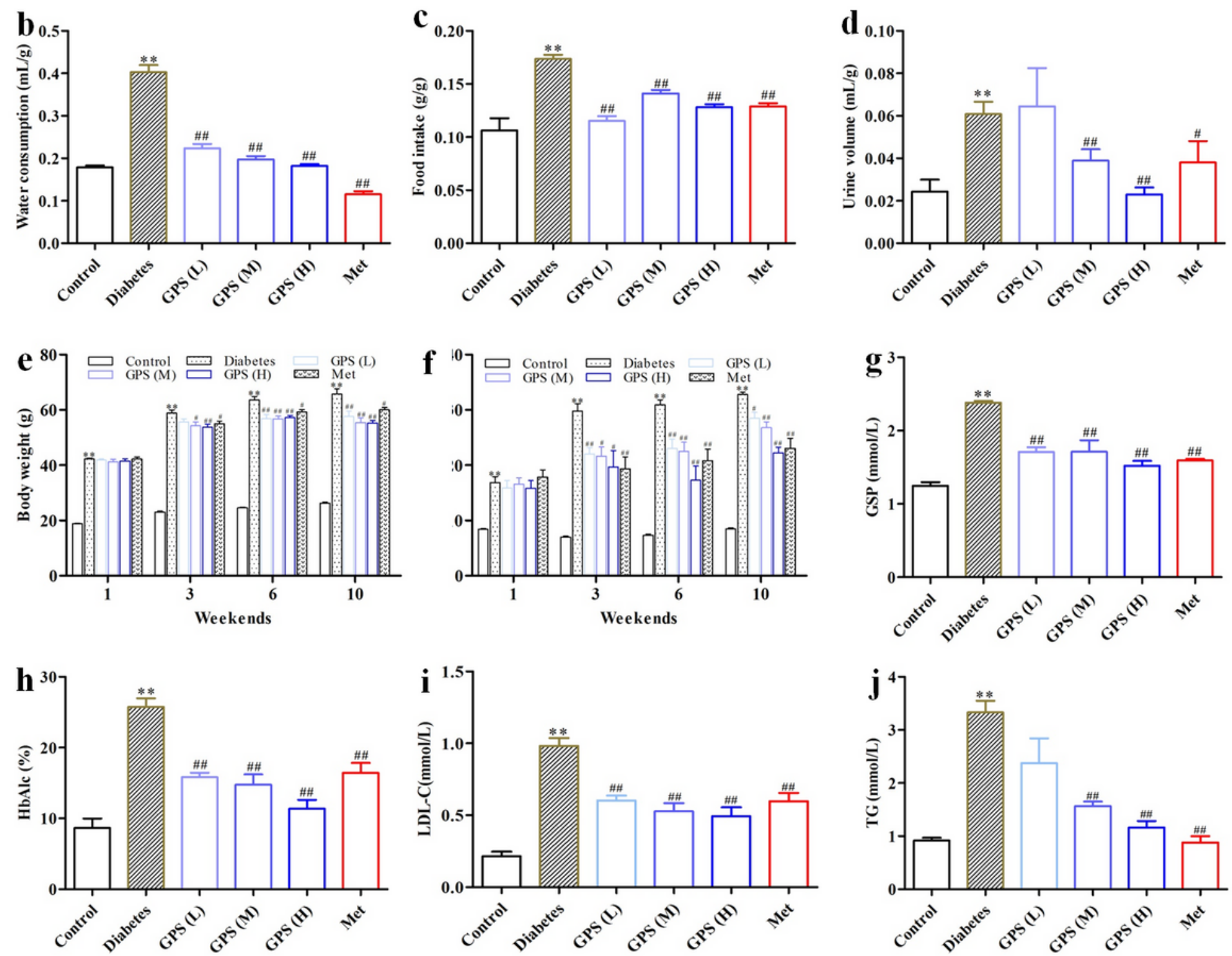

Figure 1

GPS improved metabolic parameters in $\mathrm{db} / \mathrm{db}$ mice. (a) Schematic diagram of the experiment procedure used to examine the role of GPS in diabetic mice for 10 weeks. (b-d) The water consumption, food intake and urine volume of experimental animals. (e) The body weight of experimental animals. (f-h) Serum levels of FBG, GSP and HbAlc in the experimental animals. (i-j) Serum levels of LDL-C and TG in the experimental animals. Data were expressed as means $\pm S D, n=8$. Diabetes: diabetes group, GPS $(L)$ : GPS 
treatment group (low dose: $50 \mathrm{mg} / \mathrm{kg}$ ); GPS (M): GPS treatment group (medium dose: $100 \mathrm{mg} / \mathrm{kg}$ ); GPS $(\mathrm{H})$ : GPS treatment group (high dose: $200 \mathrm{mg} / \mathrm{kg}$ ); Met: Metformin treatment group (195 mg/kg). Data was expressed as mean $\pm S D, n=8$. ${ }^{*} P<<0.01$ vs. Ctrl. \#\#P $<0.01$ and $\# P<0.05$ vs. diabetes.

a

PAS

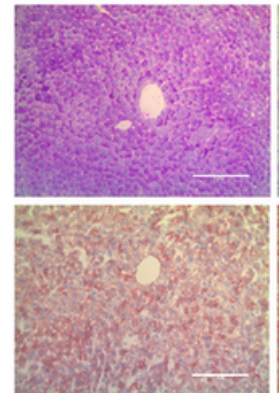

Control

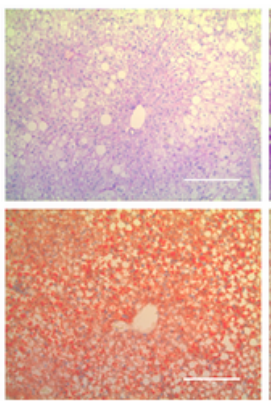

Diabetes

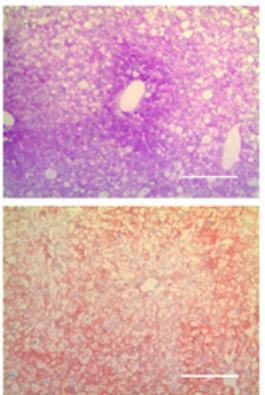

GPS (L)

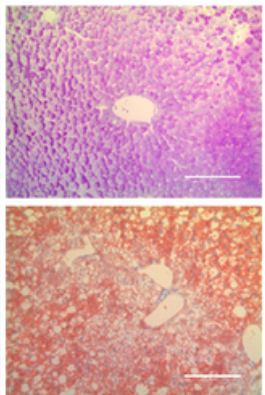

GPS (M)

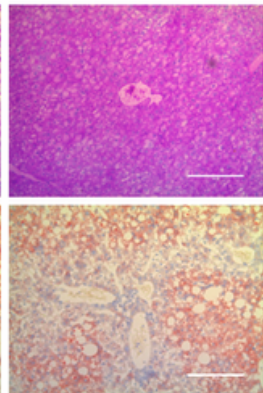

GPS (H)

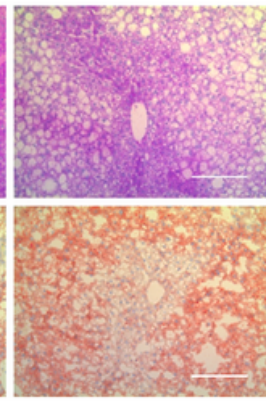

Met
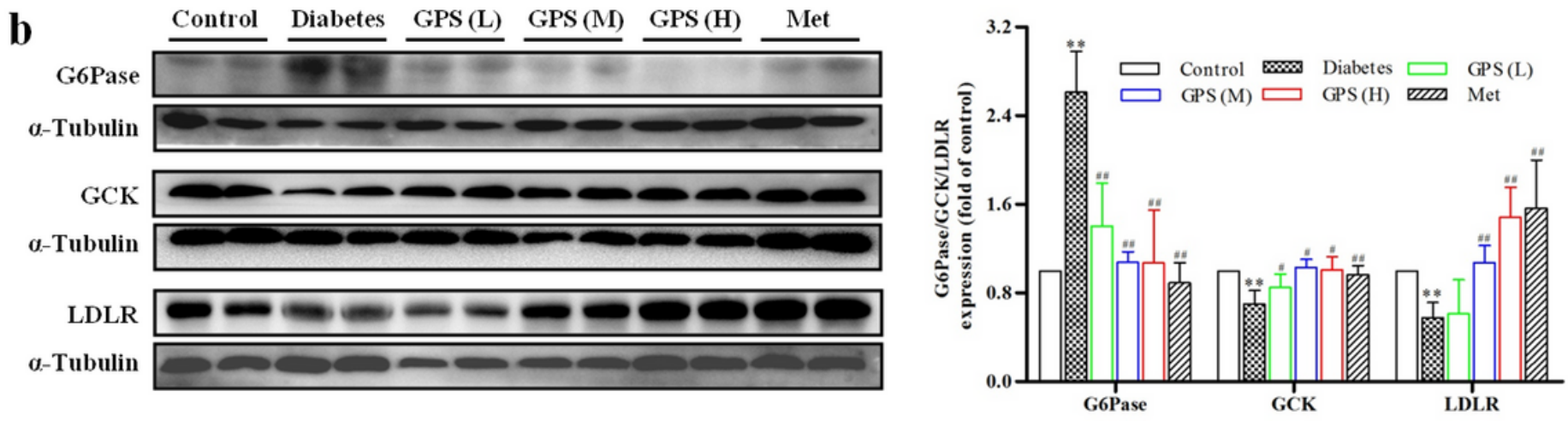

c

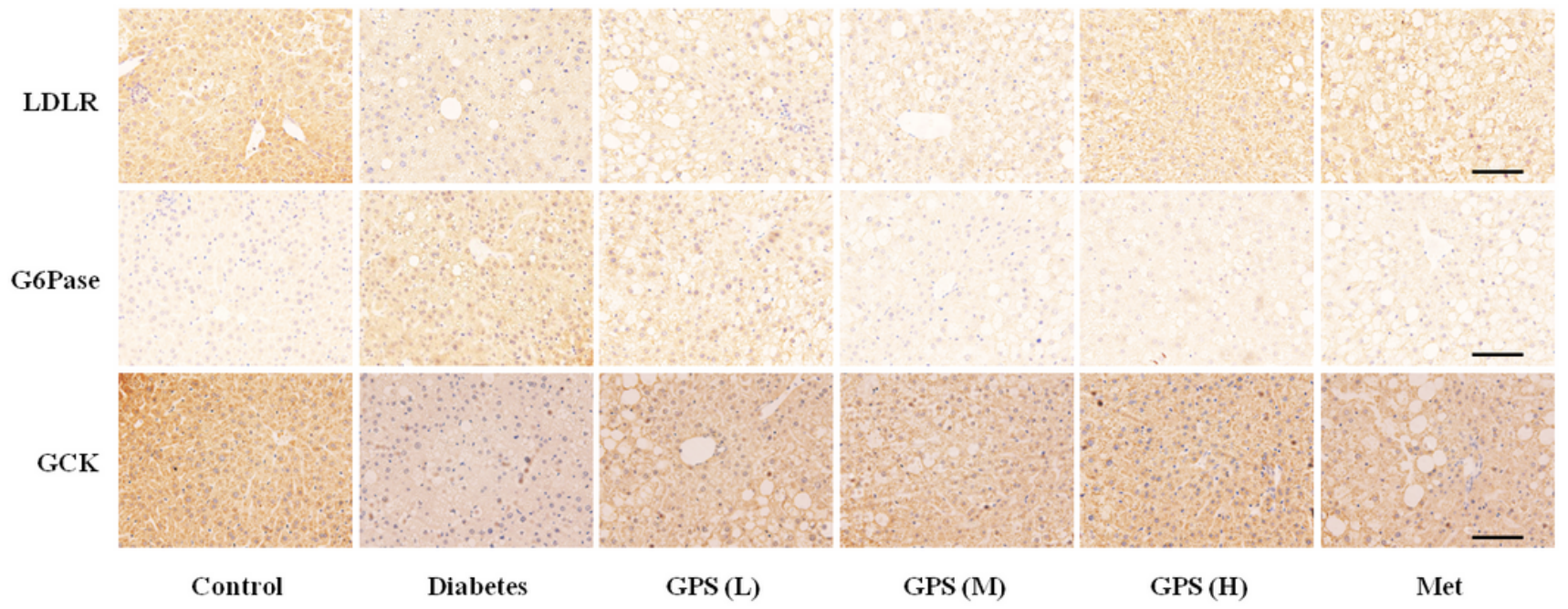

Figure 2

GPS ameliorated glucose and lipid metabolism disorders in the liver of $\mathrm{db} / \mathrm{db}$ mice. (a) The lipid deposition was detected by Oil Red 0 staining; The content of glycogen was detected by PAS staining. (b) The protein levels of G6Pase, GCK and LDLR in the liver of $\mathrm{db} / \mathrm{db}$ mice were detected by western blot. (c) The protein levels of LDLR, G6Pase and GCK in the liver of $\mathrm{db} / \mathrm{db}$ mice were detected by 
immunohistochemistry (scale bar: $200 \mu \mathrm{m}$ ). Data was expressed as mean $\pm \mathrm{SD}, \star \star P<0.01$ vs. Ctrl. \#\#P< 0.01 and \#P $<0.05$ vs. diabetes.
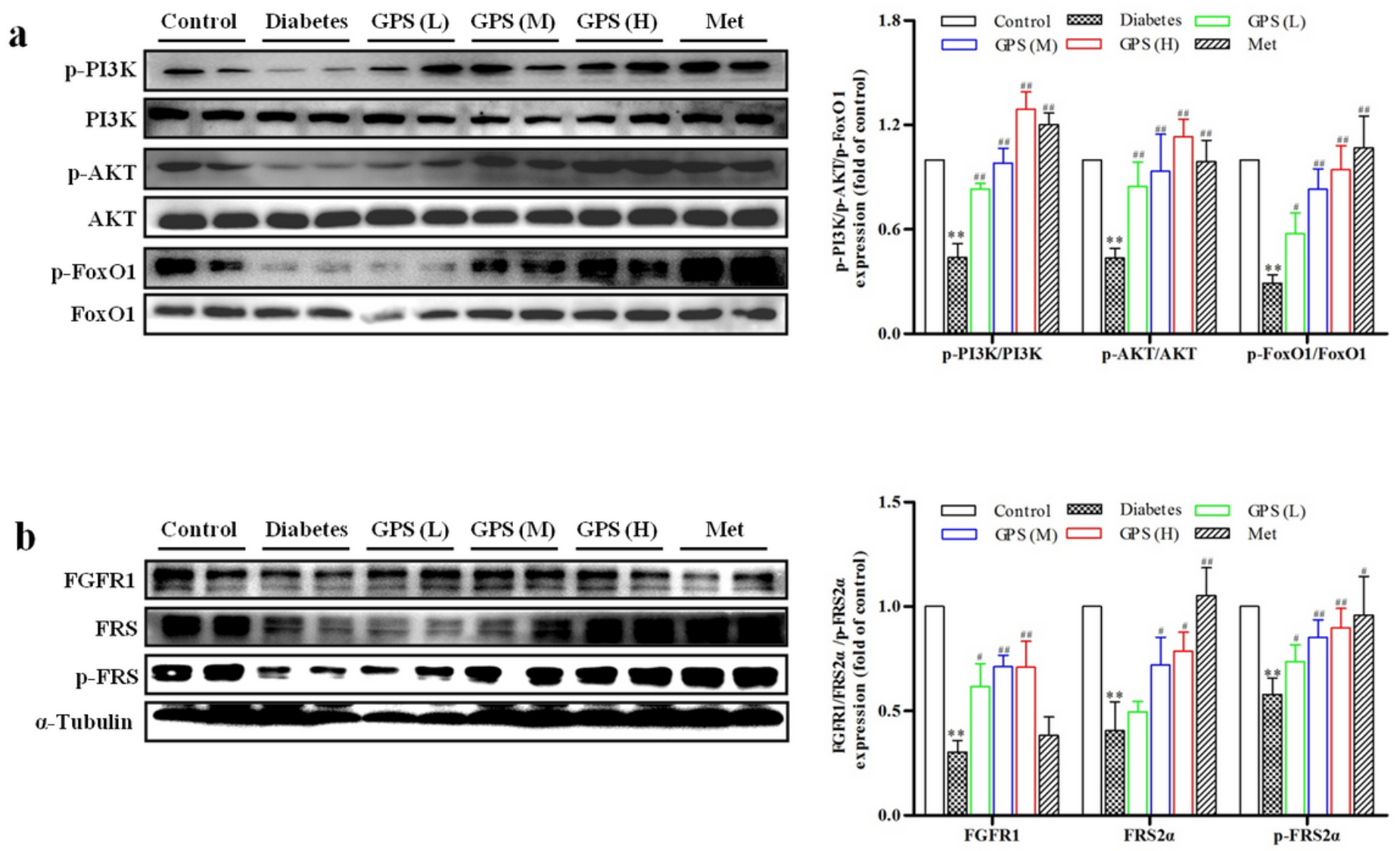

c

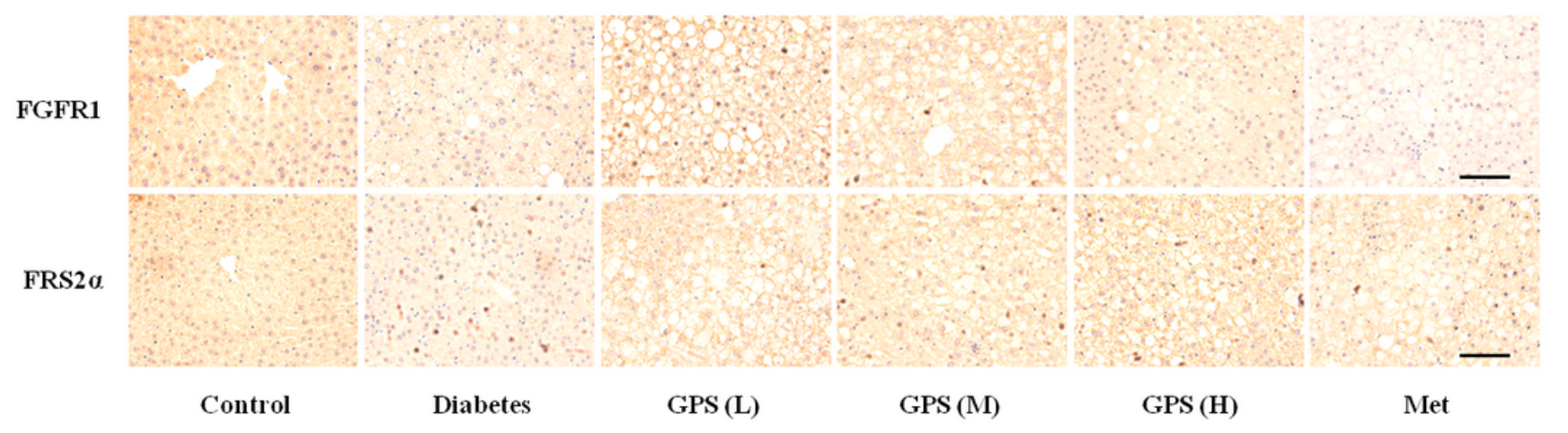

Figure 3

GPS activated FGFR1/PI3K/AKT pathway in the liver of $\mathrm{db} / \mathrm{db}$ mice. (a) The protein levels of $\mathrm{p}$-PI3K, $\mathrm{p}$-Akt and p-Fox01 in the liver of db/db mice were measured by western blot. (b) The protein levels of FGFR1, pFRS2 $a$ and FRS2 $a$ in the liver of $\mathrm{db} / \mathrm{db}$ mice were measured by western blot. (c) The protein levels of FGFR1 and FRS2 $a$ in the liver of db/db mice were detected by immunohistochemistry (scale bar: 200 $\mu \mathrm{m})$. Data was expressed as mean $\pm \mathrm{SD},{ }^{*} \mathrm{P}<0.01$ vs. Ctrl. \#\#P $<0.01$ and \#P $<0.05$ vs. diabetes. 

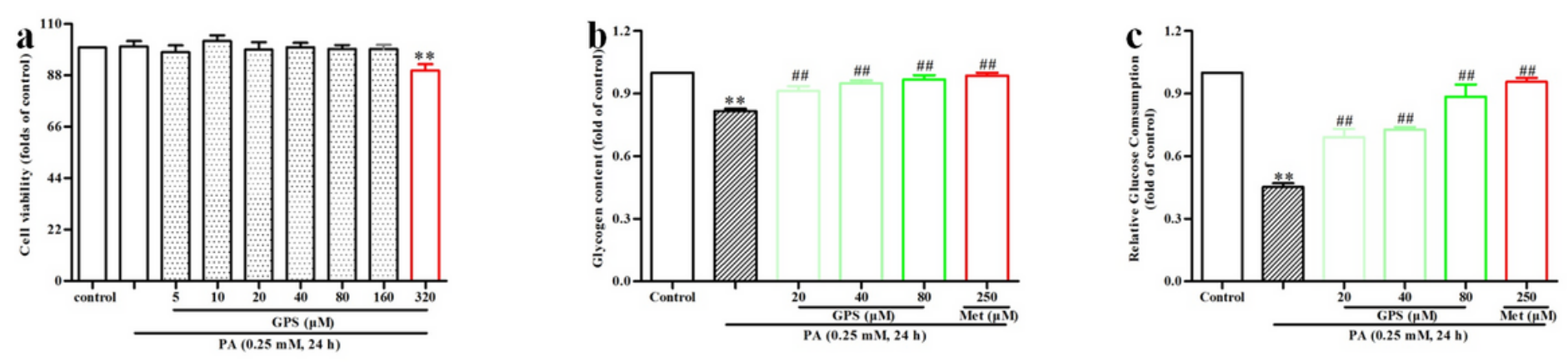

d

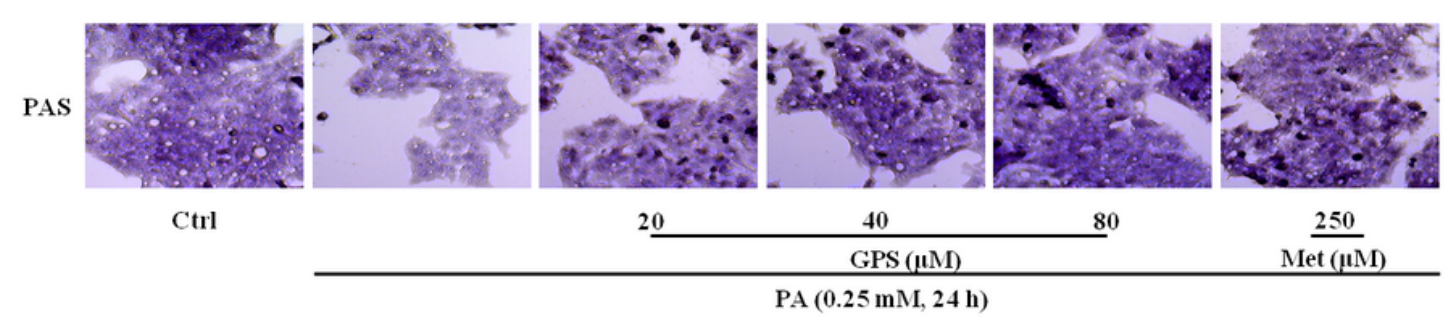

e

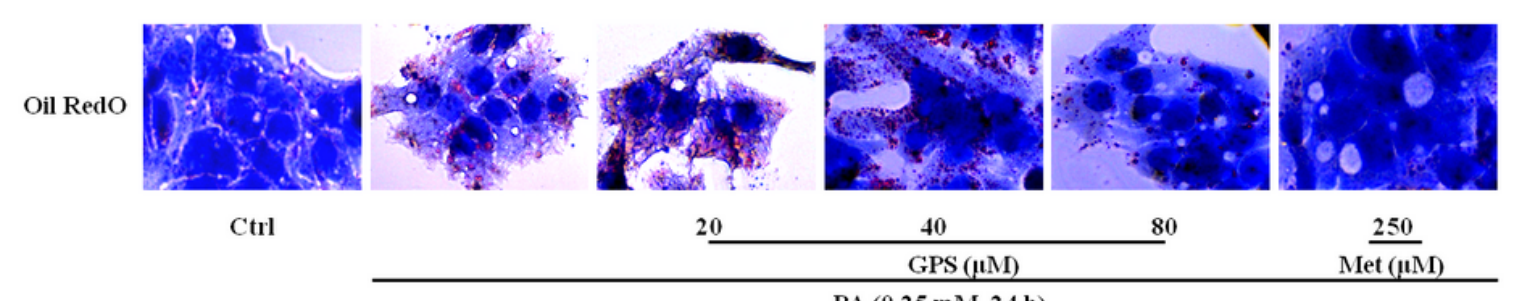

PA $(0.25 \mathrm{mM}, 24 \mathrm{~h})$

f

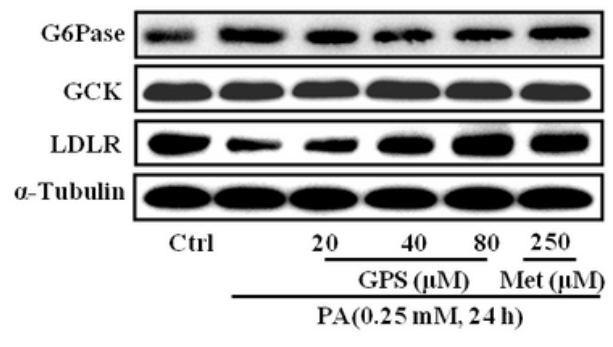

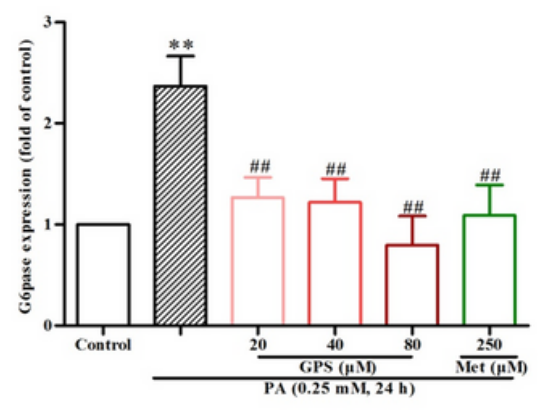

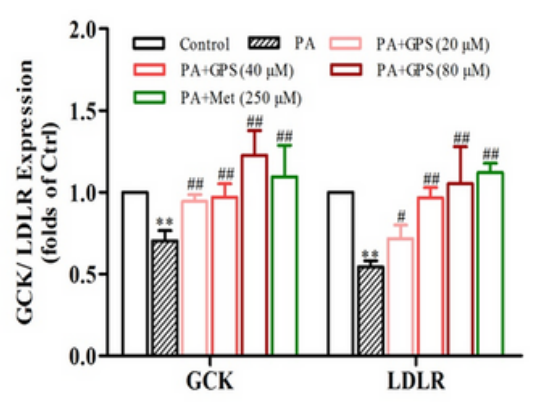

Figure 4

GPS ameliorated glucose and lipid metabolism disorders in PA-induced HepG2 cells (a) The cell viability was detected by MTT assay. (b) The effect of GPS on glycogen accumulation in PA-induced HepG2 cells was detected by test kit. (c) The effect of GPS on glucose consumption in PA-induced HepG2 cells was detected by test kit. (d) The effect of GPS on glycogen accumulation in PA-induced HepG2 cells was detected by PAS staining. (e) The effect of GPS on lipid accumulation in PA-induced HepG2 cells was detected by oil red $\mathrm{O}$ staining. (f) The effects of GPS on G6Pase, GCK and LDLR protein expressions were detected by western blot. The data were presented as mean $\pm S D$, $\star * P<0.01$ vs. control group, $\# P<0.05$ and \#\#P<0.01 vs. PA-induced alone group. 
a

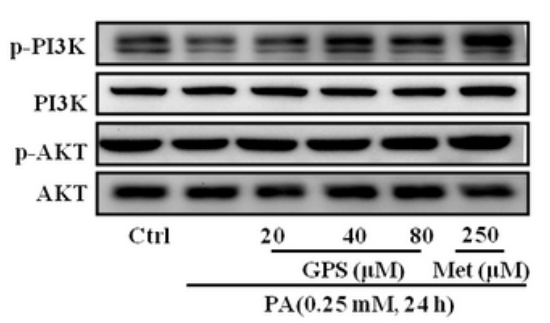

b

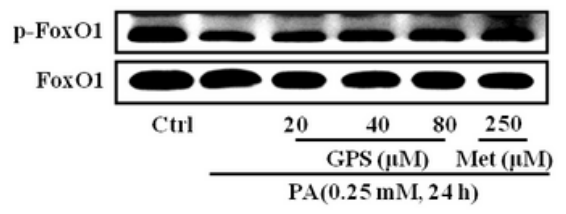

c

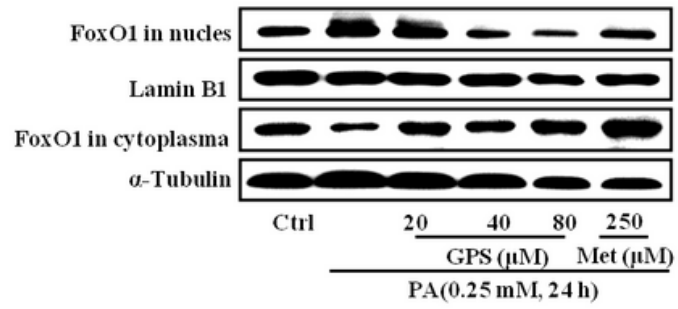

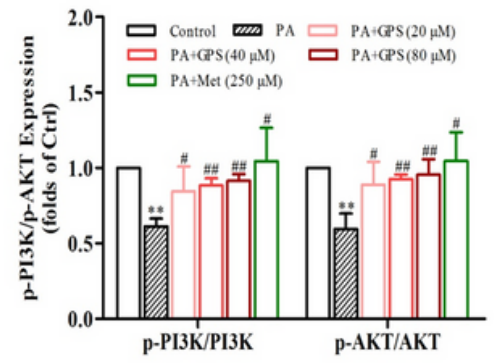
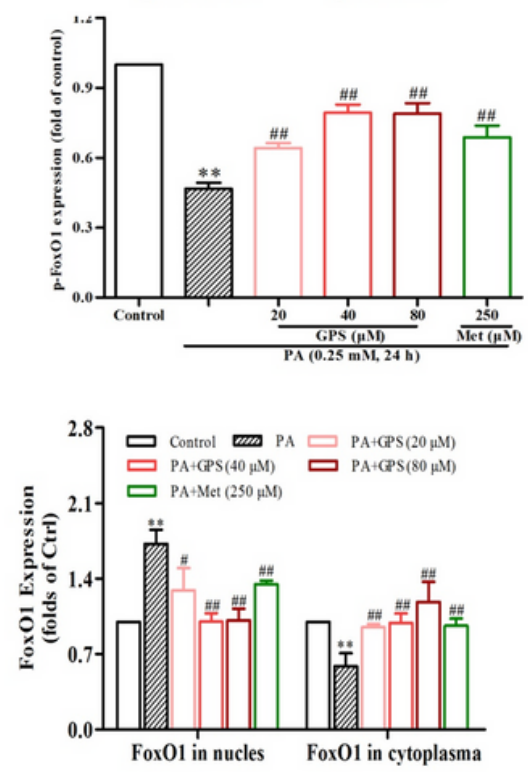

d
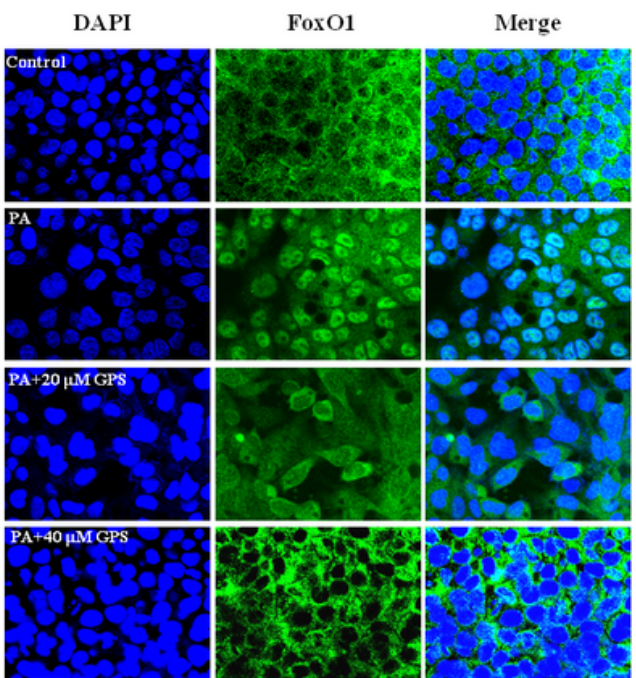

$\mathrm{PA}+80 \mu \mathrm{M}$ GPS
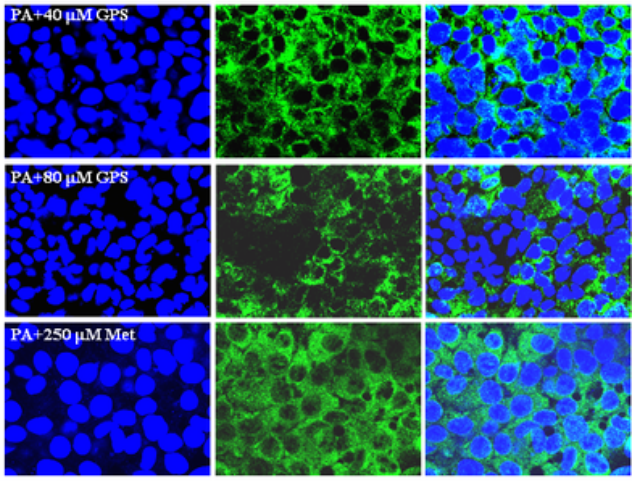

\section{Figure 5}

GPS activated PI3K/AKT pathway in PA-induced HepG2 cells. (a) The effects of GPS on p-PI3K and pAKT levels in PA-induced HepG2 cells were detected by western blot. (b) The effect of GPS on p-FoxO1 level in PA-induced HepG2 cells was detected by western blot. (c) The effects of GPS on Fox01 distribution in nucles and cytoplasma were detected by western blot. (d) The effects of GPS on Fox01 distribution in nucles and cytoplasma were detected by immunofluorescence. The data were presented as mean $\pm S D,{ }^{*} P<0.01$ vs. control group, $\# P<0.05$ and $\# \# P<0.01$ vs. $P A$-induced alone group. 
a
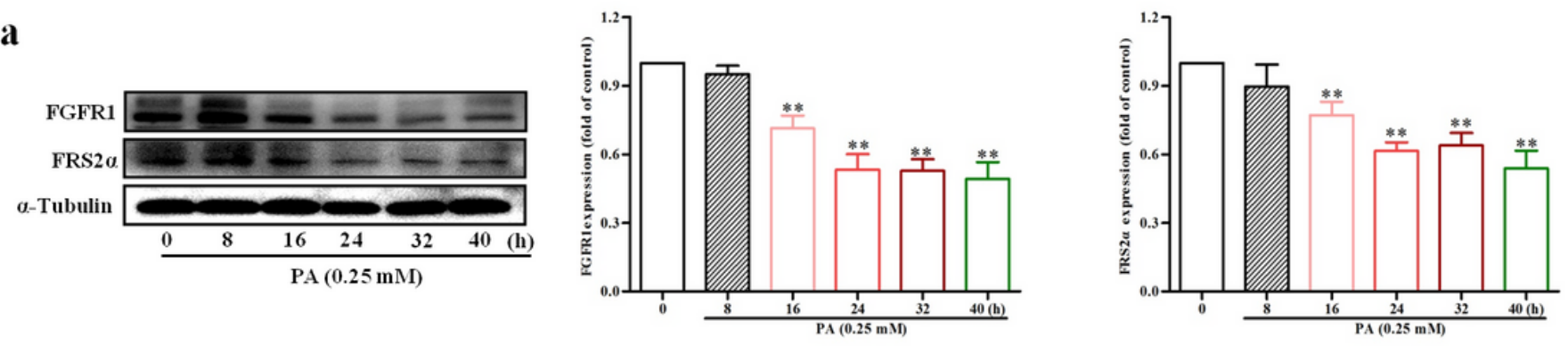

b

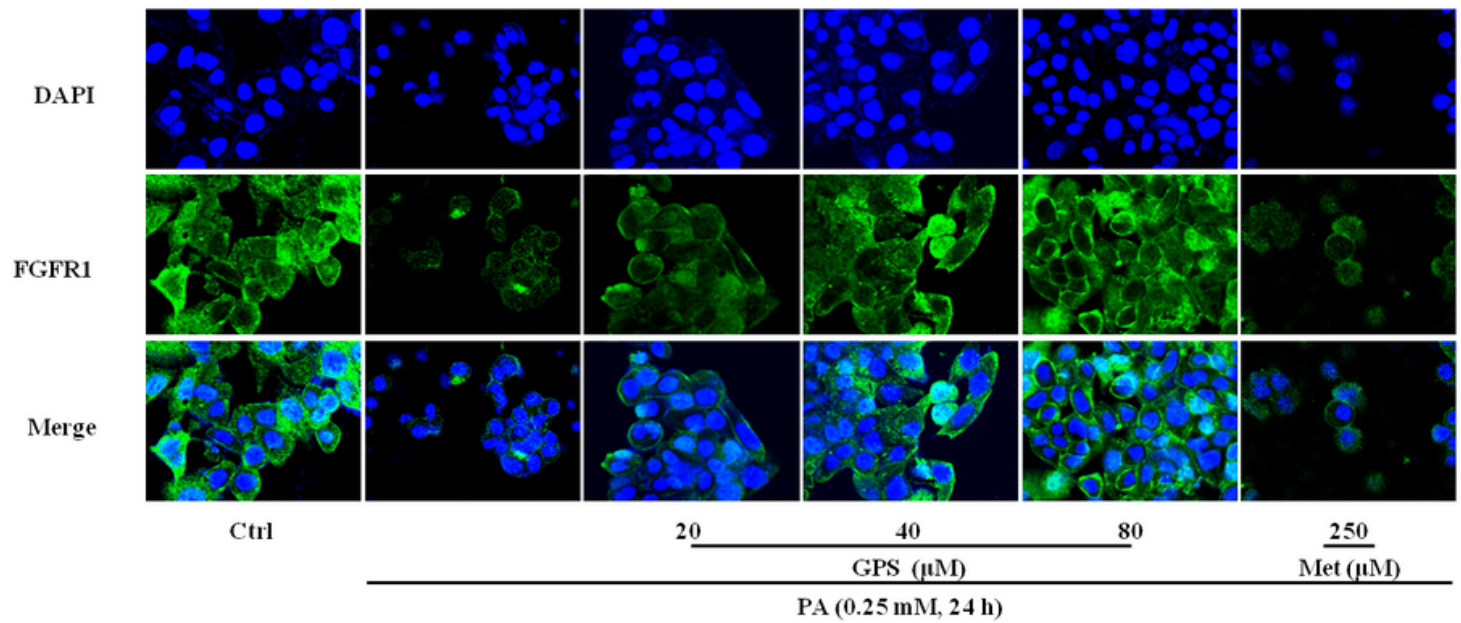

c

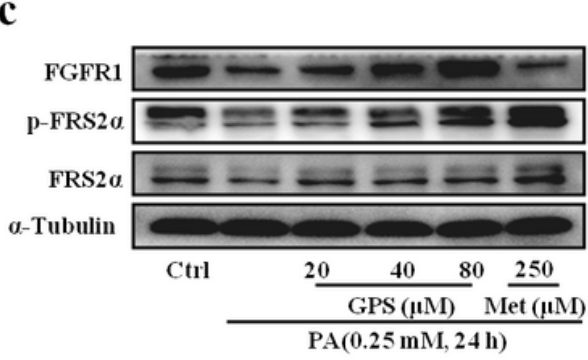

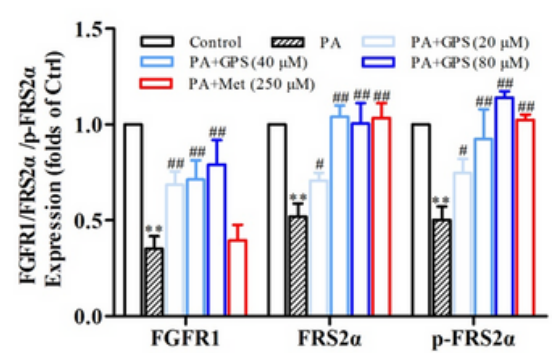

d

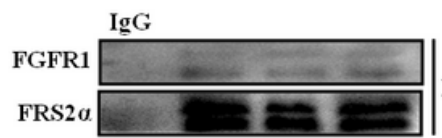
IP :FGFR1

\section{Figure 6}

GPS up-regulated FGFR1 protein expression in PA-induced HepG2 cells. (a) The protein levels of FGFR1 and FRS2 $a$ under PA stimulation at various times were detected by western blot. ${ }^{*} \mathrm{P}<0.01$ and ${ }^{*} \mathrm{P}<0.05$ vs. 0 h. (b) The effect of GPS on FGFR1 protein expression in PA-induced HepG2 cells was detected by immunofluorescence. (c) The effects of GPS on FGFR1, p-FRS2 $a$ and FRS2 $a$ protein expressions in PAinduced HepG2 cells were detected by western blot. (d) The effects of GPS on the combination of FGFR1 with FRS2 $a$ was detected by immunoprecipitation. The data were presented as mean $\pm S D$, ${ }^{\star \star} P<0.01$ vs. control group, $\# \mathrm{P}<0.05$ and $\# \# \mathrm{P}<0.01$ vs. $\mathrm{PA}$-induced alone group. 
a

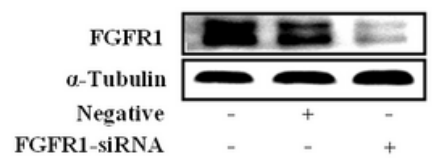

c

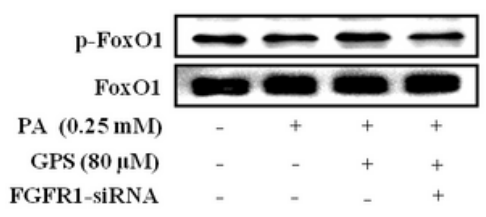

d

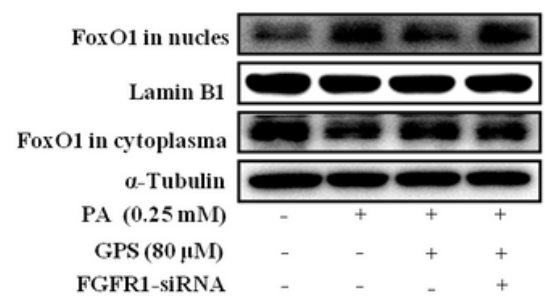

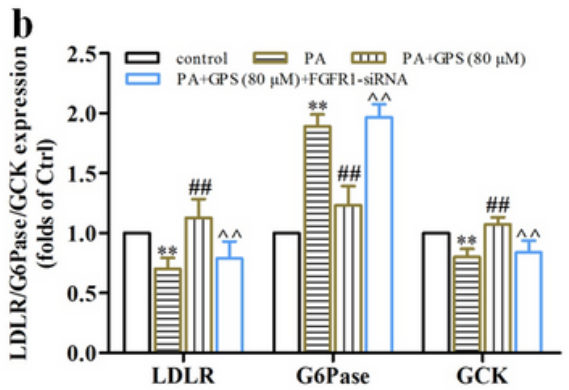
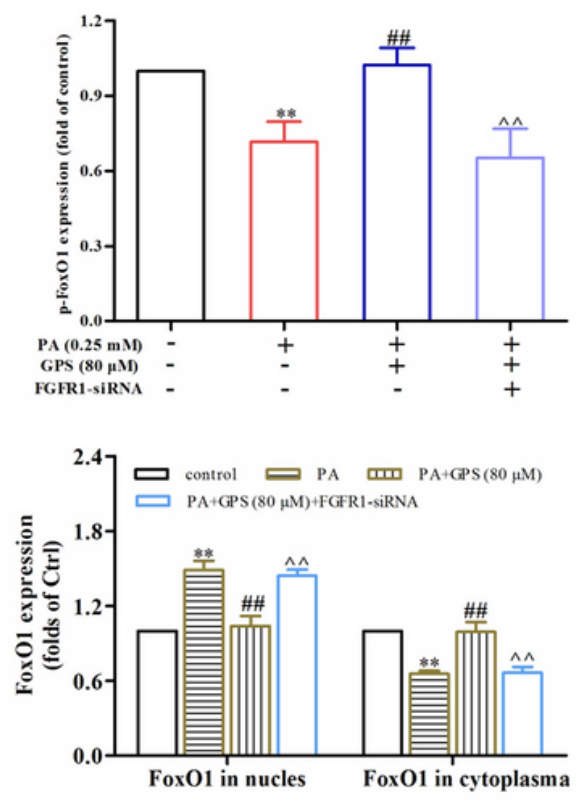

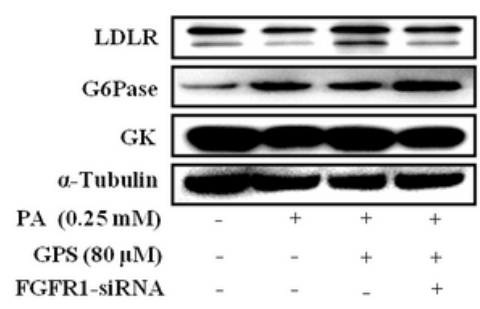

$\mathbf{e}$ DAPI

FoxO1
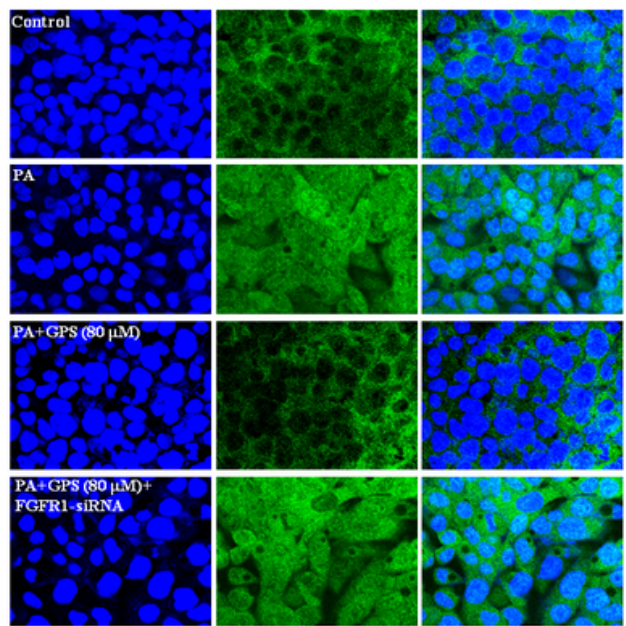

\section{Figure 7}

Knockdown of FGFR1 reversed the effects of GPS on glucose and lipid metabolism disorders in PAinduced HepG2 cells (a) The effect of FGFR1-siRNA on FGFR1 protein expression in HepG2 cells was detected by western blot. (b) The effects of GPS on G6Pase, GCK and LDLR protein expressions after the knockdown of FGFR1 were detected by western blot. (c) The effect of GPS on p-Fox01 level after the knockdown of FGFR1 was detected by western blot. (d) The effects of GPS on Fox01 distribution in nucles and cytoplasma after the knockdown of FGFR1 were detected by western blot. (e) The effects of GPS on Fox01 distribution in nucles and cytoplasma after the knockdown of FGFR1 were detected by immunofluorescence. The data were presented as mean $\pm S D,{ }^{*} P<0.01$ vs. control group, \#\#P<0.01 vs. PA-induced alone group, ${ }^{\wedge} \mathrm{P}<0.01$ vs. PA-induced+FGFR1-siRNA group. 
a

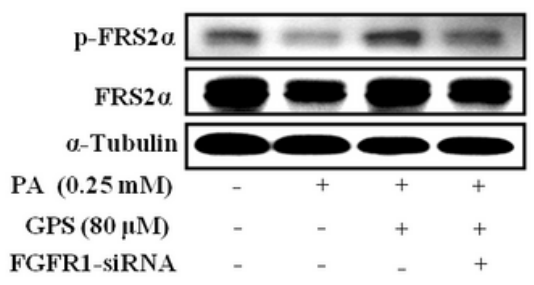

b

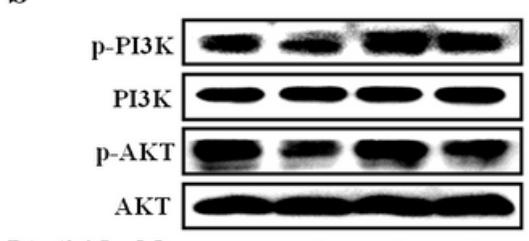

PA $(0.25 \mathrm{mM})$

GPS $(80 \mu \mathrm{M})$

FGFR1-siRNA
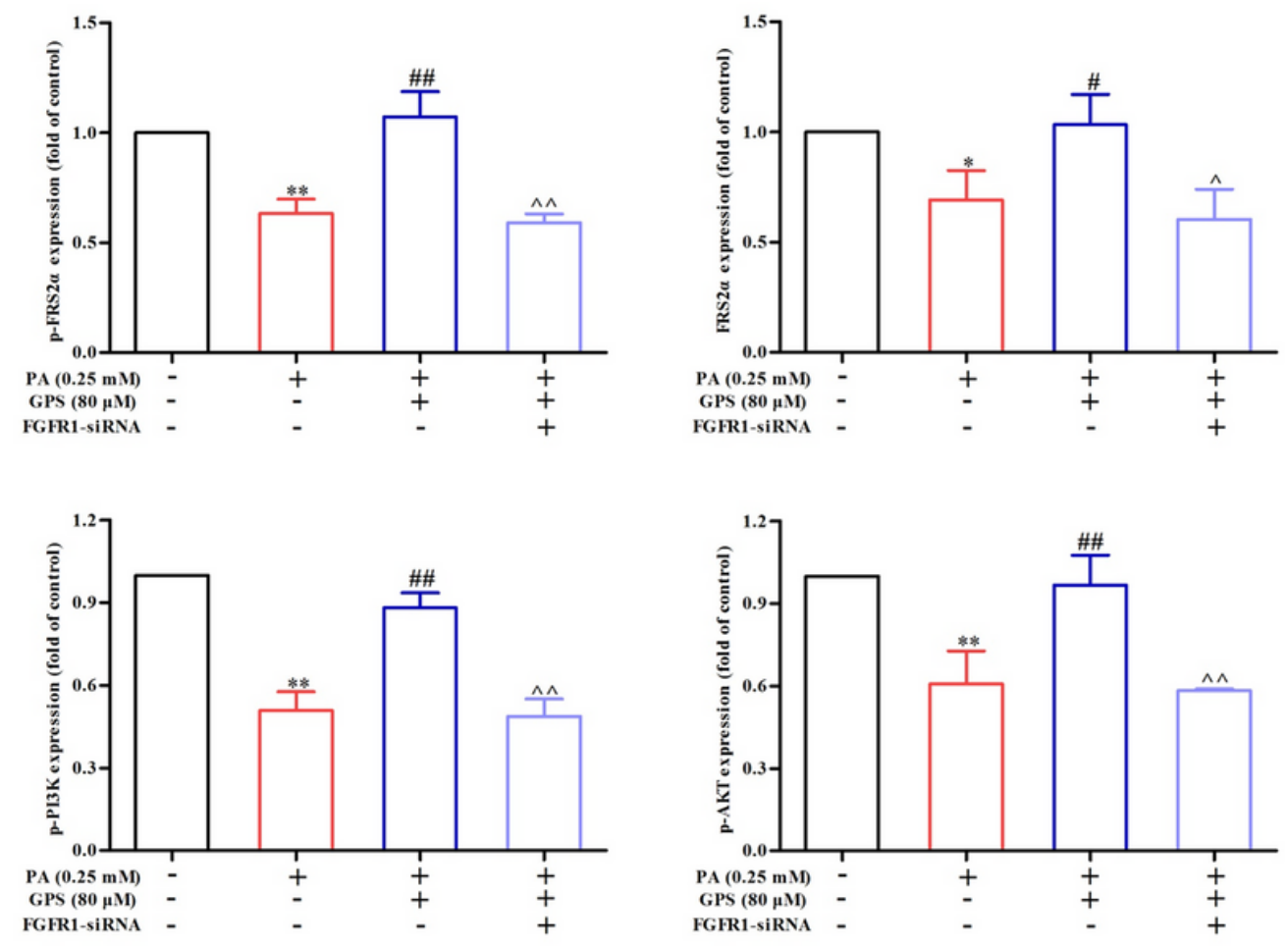

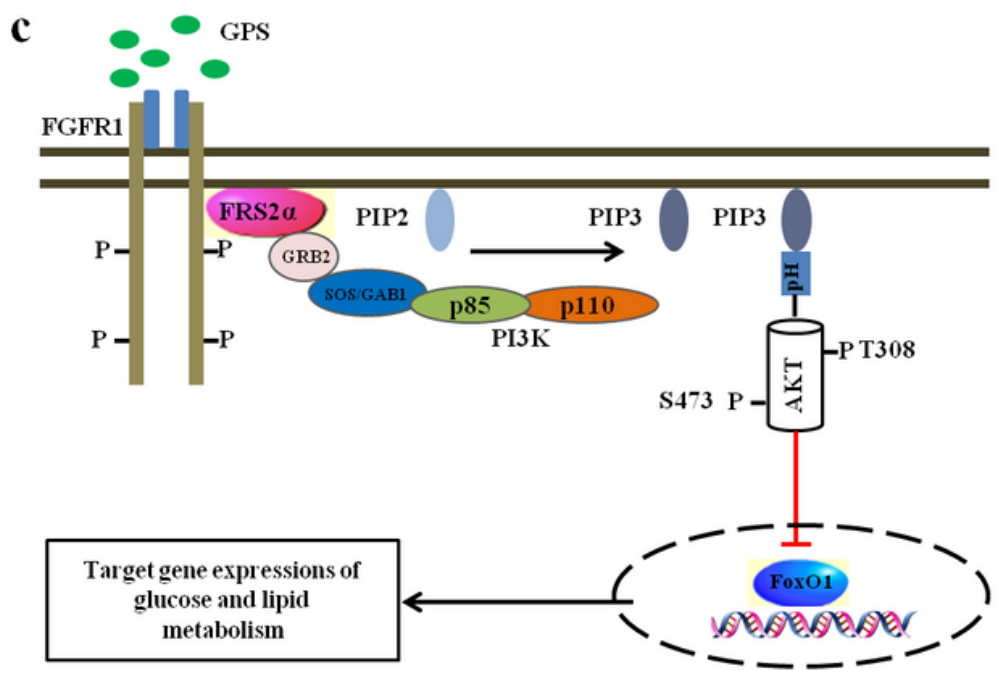

Figure 8

Knockdown of FGFR1 reversed the effects of GPS on PI3K/AKT pathway in PA-induced HepG2 cells (a) The effects of GPS on p-FRS2 $a$ and FRS2a protein expressions after the knockdown of FGFR1 were detected by western blot. (b) The effects of GPS on p-PI3K and p-AKT protein expressions after the knockdown of FGFR1 were detected by western blot. (c) Gentiopicroside ameliorates glucose and lipid metabolism disorders in T2DM by activating FGFR1/PI3K/AKT pathway. The data were presented as mean $\pm S D,{ }^{\star \star} P<0.01$ vs. control group, $\# \# P<0.01$ vs. $P A$-induced alone group, ${ }^{\wedge} P<0.05$ and ${ }^{\wedge}{ }^{\wedge} P<0.01$ vs. PA-induced+FGFR1-siRNA group.

\section{Supplementary Files}


This is a list of supplementary files associated with this preprint. Click to download.

- supplementaryInformation.docx 